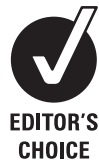

${ }^{1}$ WHO Collaborating Centre for Newborn Care and Research, Division of Neonatology, Department of Pediatrics, All India Institute of Medical Sciences, New Delhi, India; ${ }^{2}$ Department of Pediatrics, Government Medical College Hospital, Chandigarh, India

Correspondence to: Dr R K Agarwal, Division of Neonatology, Department of Pediatrics, All India Institute of Medical Sciences, New Delhi110029, India; aranag@ rediffmail.com

Accepted 27 April 2009 Published Online First 12 May 2009

\title{
Limb splinting for intravenous cannulae in neonates: a randomised controlled trial
}

\author{
S S Dalal, ${ }^{1}$ D Chawla, ${ }^{2}$ J Singh,, R K Agarwal, ${ }^{1}$ A K Deorari, ${ }^{1}$ V K Paul ${ }^{1}$
}

\section{ABSTRACT}

Objective: To evaluate the efficacy of peripheral intravenous (IV) cannula site joint immobilisation by splint application on functional duration of peripheral IV cannula in neonates.

Design: Randomised controlled trial.

Setting: Neonatal intensive care unit of a tertiary care hospital.

Participants: Neonates requiring continuous IV infusion for an expected duration of more than or equal to 72 hours.

Intervention: Eligible cannulations were randomised to either "splint" or "no-splint" group. In the splint group, a cardboard splint was used to immobilise the joint at peripheral IV cannula site. No attempt was made to immobilise the limb in the no-splint group.

Outcome measure: Functional duration of a peripheral IV cannula measured as interval from time of insertion to the development of predefined sign of removal (extravasation, blockage, inflammation).

Results: A total of 69 peripheral IV cannulations in 54 neonates were randomised to either the splint $(n=33)$ or no-splint group $(n=36)$. Both groups were comparable in birth weight, gestation, site of cannulation and nature of fluids administered. Mean functional duration of cannula was lesser in the splint group compared to the no-splint group (h; 23.5 (SD15.9) vs 26.9 (SD15.5), mean difference: $-3.3 \mathrm{~h}, 95 \% \mathrm{Cl}-11.02$ to $4.3 \mathrm{~h}$ ) although the difference was not statistically significant $(p=0.38)$. Extravasation at cannula site was found be the commonest indication for cannula removal in both the groups ( $84 \%$ vs $76.5 \%)$.

Conclusion: Joint immobilisation with splint at cannula site did not improve the functional duration of peripheral IV cannula.

Neonates admitted to neonatal intensive care unit (NICU) routinely undergo peripheral venous cannulation for administration of intravenous (IV) fluids and drugs. This often requires multiple peripheral venous cannulations to provide fluids and drugs for prolonged periods. Multiple cannulations expose the vulnerable newborns to repeated painful and stressful experiences as well as the risk of infection, which might result in adverse effect on health and long-term neurodevelopmental outcome. ${ }^{1}$ Measures to prolong the functional duration of a peripheral IV cannula may reduce the number of cannulation attempts and save neonates from pain and stress, as well as reduce the risk of infection. Newer cannulae are soft, flexible and easy to insert and last longer compared to scalp vein needles used in the past. ${ }^{2}$ Polytetrafluorethylene catheters are found to remain functional three times longer than steel

\section{What is already known on this topic}

- Splinting a peripheral cannula that overlies a joint is a common practice.

- The efficacy of this practice has never been formally tested.

\section{What this study adds}

- There is no role for immobilising the limb with a splint to prolong the functional duration of peripheral intravenous cannulae in neonates.

needles used in the past with no apparent increase in complications. ${ }^{3}$ Splinting to immobilise the limb is one traditionally used method to prolong the functional duration of these cannulae. It is not known whether the application of a splint is of any benefit in prolonging the functional duration of these newer polytetrafluorethylene cannulae in newborn populations. The present study was designed to study the efficacy of splinting the joint on the functional duration of peripheral IV cannula in neonates requiring continuous IV infusion.

We hypothesised that in neonates, splinting to immobilise the limb at cannulation site would prolong the functional duration of peripheral IV cannula as compared to no immobilisation.

\section{METHODS AND SETTING}

Study was conducted as a randomised controlled trial in neonatal intensive care unit (NICU) of a tertiary care hospital. All preterm and term neonates admitted in the NICU and anticipated to require peripheral continuous IV infusion for an expected period of $\geqslant 72$ hours were eligible for enrolment into the study. Neonates with major congenital malformations were excluded. An eligible cannulation was defined as one of the first three successful cannulations performed in eligible neonates with cannula being inserted over one of the four major joints, that is, wrist, elbow, knee or ankle. Eligible and successful cannulations were randomised to "splint" or "no-splint" groups. A neonate could be enrolled more than once with each eligible cannulation being randomised independent of group allocation of previous cannulation. Allocation of group was done by randomisation. A random number sequence was generated in a fixed block size of four each using a 
web-based random number generator. The random codes were kept in serially numbered, opaque and sealed, identical envelopes.

Identical procedure for cannula insertion and fixation was used in both of the groups. Senior (neonatology training fellows) and junior (paediatric residents) residents were responsible for IV cannulation, while nurses assisted them in fixing the cannula. Twenty-four gauge peripheral IV cannulae (BD Neoflon, Becton Dickinson India Pvt. Ltd, Haryana, India) supplied by the hospital were used for cannulation in all neonates. Prior to the onset of the study, all healthcare personnel involved in insertion and fixation of cannula in the NICU were provided with instructions on the technique of insertion and fixation of peripheral IV cannulae at various joints. In the splint group, a splint made up of cotton and gauze piece rolled over a hard cardboard piece was applied to the limb immediately after fixing the cannula, as per the standardised method, to prevent movement at the underlying joint. Dimensions of the splints used were standardised as length extending two inches on either side of the joint and width equal to the width of the limb just proximal to the joint. In the nosplint group the cannula was fixed, as per the standardised method, and no effort was made to immobilise the joint. IV fluids and drugs were administered using syringe infusion pumps. After insertion and fixation, the cannula site was monitored for development of sign of removal every 2 hours by a resident or nurse on-duty. The sign of removal was defined as presence of any one of the following:

- extravasation - characterised by swelling and oedema at the cannula site

- blockage-indicated by high occlusion on infusion pump

- inflammation - characterised by swelling, redness and raised temperature or tenderness over the cannula site.
If a baby no longer needed the cannula for a clinical indication or no longer required continuous IV fluids, time to such an event was noted. The functional duration of the cannula was measured in hours, from time of insertion to time of development of a sign of removal. As part of the clinical protocol we did not use intermittent flushes or heparin lock to maintain patency of IV cannula. We also avoided using muscle relaxants or sedatives.

Mean functional duration of the peripheral IV cannula without splint was found to be 48 hours (SD36 hours) in our NICU in a pilot study (unpublished). To detect a difference of at least $24 \mathrm{~h}$ with $80 \%$ power and $95 \%$ confidence interval (CI), we needed to enrol 36 peripheral IV cannulations. The protocol was cleared by the Institutional Ethics Committee. Informed written consent was taken from one of parents of enrolled newborns.

All baseline and outcome data were recorded prospectively in a predesigned and pretested data collection form. The data were checked for completion, consistency and accuracy. Data were analysed using the software Stata V.9.1. Group characteristics were compared with $\chi^{2}$ test and two-sample $t$ test for discrete and continuous variables respectively. A $p$ value of $<0.05$ was taken as statistically significant. Analysis was by intention to treat.

\section{RESULTS}

The study was conducted from November 2006 to July 2007. Figure 1 provides the participant flow as per CONSORT guidelines. ${ }^{4}$ A total of 387 neonates were admitted to NICU during the study period. Of these, 54 neonates were enrolled into the study and 69 cannulations performed on these neonates were included into the study. Out of the 69 cannulations enrolled, one cannulation in each group was excluded from analysis as the cannulation site was not over a joint. One
Figure 1 Study flow chart. *Up to three successful cannulations in each eligible neonate could be enrolled and randomised.

\section{Neonates admitted to NICU during study period and assessed for eligibility $(n=387)$}

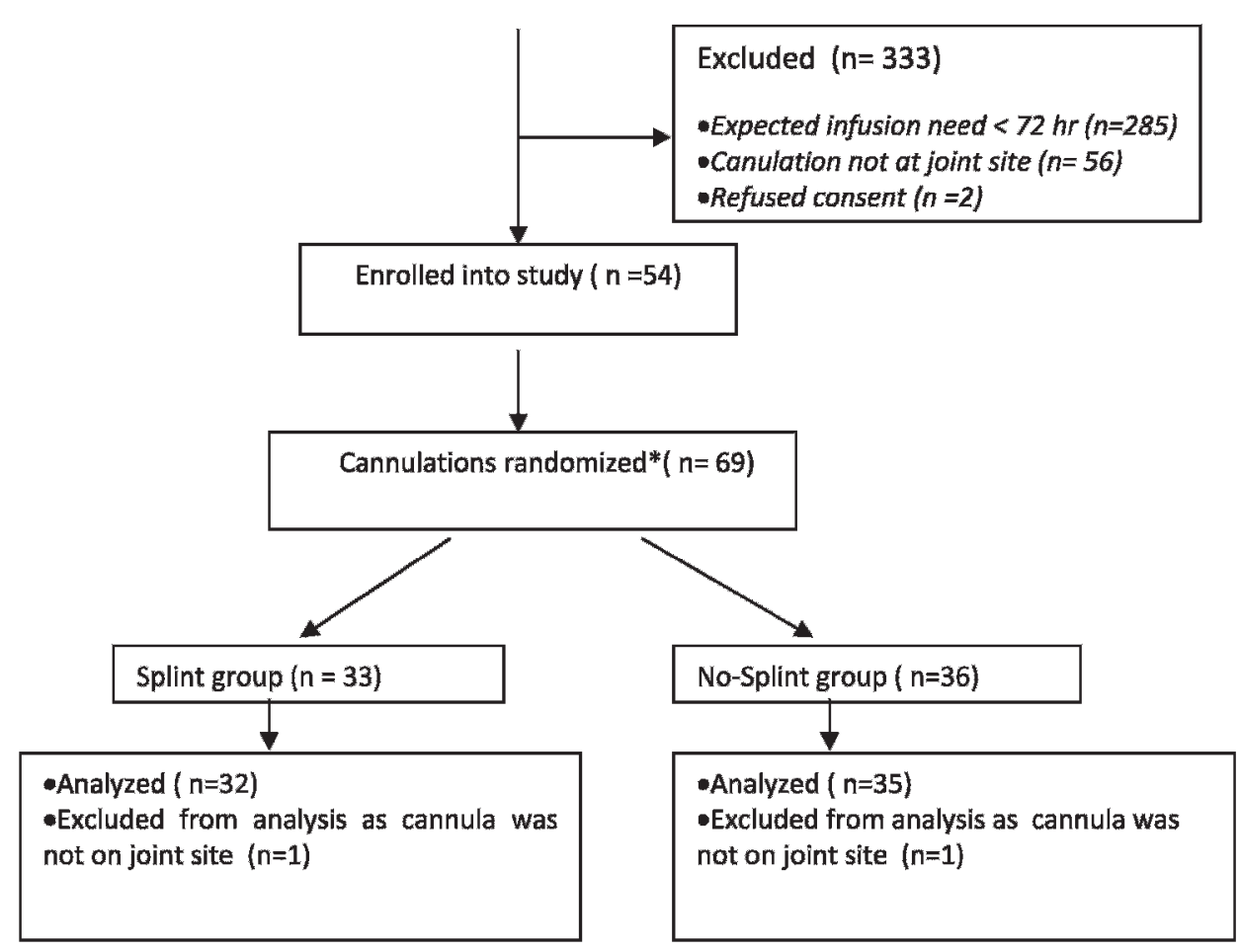


Table 1 Baseline characteristics of neonates enrolled in two groups

\begin{tabular}{|c|c|c|}
\hline Baseline variable & Splint group $(n=33)$ & No-splint group $(n=36)$ \\
\hline Gestation (wks) & $32(30-33)$ & $33(31-35)$ \\
\hline Birth weight (g) & $1226(984-1511)$ & 1131 (933-1352) \\
\hline \multicolumn{3}{|l|}{ Mode of delivery } \\
\hline Caesarean & $25(77 \%)$ & $31(86 \%)$ \\
\hline Other & $8(23 \%)$ & $5(14 \%)$ \\
\hline \multicolumn{3}{|l|}{ Site of cannulation } \\
\hline Wrist & $26(79 \%)$ & $26(74 \%)$ \\
\hline Others & $7(21 \%)$ & $10(26 \%)$ \\
\hline \multicolumn{3}{|l|}{ Cannula inserter } \\
\hline Senior resident & $19(58 \%)$ & $22(63 \%)$ \\
\hline Junior resident & $12(36 \%)$ & $13(37 \%)$ \\
\hline Nurse & $2(6 \%)$ & 0 \\
\hline \multicolumn{3}{|l|}{$\begin{array}{l}\text { Serial number of } \\
\text { cannulation }\end{array}$} \\
\hline First & $16(49 \%)$ & $25(69 \%)$ \\
\hline Second & $11(33 \%)$ & $6(17 \%)$ \\
\hline Third & $6(18 \%)$ & $5(14 \%)$ \\
\hline \multicolumn{3}{|l|}{ Type of IV fluid } \\
\hline $10 \%$ dextrose & $7(23 \%)$ & $14(40 \%)$ \\
\hline $\begin{array}{l}10 \% \text { dextrose + calcium } \\
\text { gluconate }\end{array}$ & $12(39 \%)$ & $13(37 \%)$ \\
\hline $\mathrm{N} / 5$ in $10 \%$ dextrose & $1(3 \%)$ & 0 \\
\hline Parenteral nutrition & $9(29 \%)$ & $6(17 \%)$ \\
\hline Others & $2(6.5 \%)$ & $2(6 \%)$ \\
\hline
\end{tabular}

Data expressed as median (inter-quartile range) or $\mathrm{n}(\%)$.

neonate in the no-splint group was transferred out of NICU before development of signs of removal and could not be monitored further. The cannula was functional until 54.5 hours after insertion.

The groups were comparable with respect to gestation, birth weight, mode of delivery and the site of cannulation (table 1). There was no difference between the groups in the nature of fluid administered, person inserting the cannula and serial number of cannulation randomised to the group.

Mean functional duration of the peripheral IV cannula was less in the splint group compared to the no-splint group, although the difference was not statistically significant (hours; 23.5 (SD5.9) vs 26.9 (SD15.5); mean difference $-3.3,95 \%$ CI: -11.0 to $4.3 ; p=0.38$ ). This difference was more in neonates with gestational age $<30$ wks, however the sample size in this group of neonate is too small to arrive at any conclusion. Extravasation at cannula site was the most common cause of cannula removal in both the groups ( $84 \%$ vs $76.5 \%$ of cases). Complications like skin damage and allergic reaction related to use of splint were not observed.

\section{DISCUSSION}

The usefulness of splint application to immobilise the joint after peripheral IV cannula insertion has remained unresolved as this issue has never been studied in a controlled trial. The present study, therefore, attempts to resolve the issue of the usefulness of splint in prolonging the functional duration of a peripherally inserted IV cannula through a randomised controlled trial. Johnson et al ${ }^{5}$ surveyed prospectively peripheral IV cannula use in a neonatal intensive care unit to ascertain the rate of complications and the factors influencing the life span of an IV cannula. During a three-month period in which 199 IV cannulae were inserted in 69 patients, only two significant infiltrations (1\%) were observed in more than 5000 hours of IV therapy. None of the factors studied, including weight, age, type and rate of fluid administration, and type of medication (except pancuronium bromide), had any discernible effect on the functional life span of IV cannulae.

In another similar study Gupta et $a l^{6}$ studied variables affecting life span of a peripheral IV cannula in a NICU of a developing country. The median survival time of an IV cannula, as expressed by Kaplan-Meir survival analysis, was 40 hours (SE, 2.49; 95\% CI, 35.12 to 44.88). Birth weight, gestation, application of splint, fluid and glucose infusion rate, site of cannulation and administration of antibiotics (ampicillin, gentamicin, amikacin, vancomycin) phenobarbitone, blood products, or calcium gluconate did not influence the median life span of IV cannulae.

In present study, mean functional duration of peripheral IV cannulae in both groups was less than as reported by Gupta et al. ${ }^{6}$ This is probably due to the enrolment of a greater number of premature babies in the present study. Splint application was found to be associated with a decrease in functional duration of a peripheral IV cannula, although the difference was not statistically significant. This effect is more pronounced in neonates with lower gestational age (gestational age $<30$ weeks); however, the number of subjects in this group was too small to draw any conclusion. Extravasation at the cannula site is found to be the commonest indication for cannula removal. From these observations it can be hypothesised that splint application may promote extravasation by causing pressure on the draining veins via the adhesive tape used for fixing the splint proximally. Infants with a lower gestational age have immature thin walled veins with poor vascular tone, predisposing them to greater risk of extravasation even with slight external pressure. The type of fluid administered may also affect the rate of extravasation. Irritant fluids may cause early extravasations, however, there was no difference in the nature of fluid administered in the two groups. Also, previous studies have found no effect of type and rate of fluid administration on functional life span of the peripheral IV cannula. ${ }^{56}$ The present study addresses a common practice issue through a randomisation trial in NICU setting. Blinding of the observers monitoring for signs of removal was not possible due to the nature of the intervention, which might have introduced some bias.

In conclusion, it can be stated that application of a splint to immobilise the joint while using a peripheral IV cannula does not prolong the functional duration of the cannula.

Competing interests: None.

Ethics approval: Ethics committee approval was obtained from Ethics Committee, All India Institute of Medical Sciences, New Delhi, India.

Provenance and peer review: Not commissioned; externally peer reviewed.

\section{REFERENCES}

1. Johnston CC, Stevens BJ. Experience in a neonatal intensive care unit affects pain response. Pediatrics 1996;98:925-30

2. Mauricio R, Barria P, Gemasantander M. Peripheral vascular access in newborn of intensive care unit: experience of a public hospital. Ciencia Y Enfermeria 2006;XII:35-44

3. Batton DG, Maisels MJ, Appelbaum P. Use of peripheral intravenous cannulas in premature infants: a no-splinted study. Pediatrics 1982;70:487-90.

4. CONSORT. See http://www.consort-statement.org/home/ (accessed 8 Jul 2009).

5. Johnson RV, Donn SM. Life span of intravenous cannulas in a neonatal intensive care unit. Am J Dis Child 1988;142:968

6. Gupta $\mathbf{P}$, Rai R, Basu $\mathrm{S}$, et al. Life span of peripheral intravenous cannula in a neonatal intensive care unit of a developing country. J Pediatr Nurs 2003;18:287-92. 